\title{
Layered smooth muscle cell-endothelial progenitor cell sheets derived from the bone marrow augment postinfarction ventricular function
}

\author{
Yasuhiro Shudo, MD, PhD, ${ }^{a}$ Andrew B. Goldstone, MD, PhD, ${ }^{a}$ Jeffrey E. Cohen, MD, ${ }^{\mathrm{a}}$ Jay B. Patel, BS, \\ Michael S. Hopkins, BS, ${ }^{a}$ Amanda N. Steele, BS, ${ }^{a}$ Bryan B. Edwards, BE, ${ }^{a}$ Masashi Kawamura, MD, PhD, ${ }^{a}$ \\ Shigeru Miyagawa, MD, PhD, ${ }^{b}$ Yoshiki Sawa, MD, PhD, ${ }^{b}$ and Y. Joseph Woo, MD
}

\section{ABSTRACT}

Objective: The angiogenic potential of endothelial progenitor cells (EPCs) may be limited by the absence of their natural biologic foundation, namely smooth muscle pericytes. We hypothesized that joint delivery of EPCs and smooth muscle cells (SMCs) in a novel, totally bone marrow-derived cell sheet will mimic the native architecture of a mature blood vessel and act as an angiogenic construct to limit post infarction ventricular remodeling.

Methods: Primary EPCs and mesenchymal stem cells were isolated from bone marrow of Wistar rats. Mesenchymal stem cells were transdifferentiated into SMCs by culture on fibronectin-coated culture dishes. Confluent SMCs topped with confluent EPCs were detached from an Upcell dish to create a SMC-EPC bi-level cell sheet. A rodent model of ischemic cardiomyopathy was then created by ligating the left anterior descending artery. Rats were randomized into 3 groups: cell sheet transplantation $(n=9)$, no treatment $(n=12)$, or sham surgery control $(\mathrm{n}=7)$.

Results: Four weeks postinfarction, mature vessel density tended to increase in cell sheet-treated animals compared with controls. Cell sheet therapy significantly attenuated the extent of cardiac fibrosis compared with that of the untreated group (untreated vs cell sheet, 198 degrees [interquartile range (IQR), 151-246 degrees] vs 103 degrees [IQR, 92-113 degrees], $P=.04$ ). Furthermore, EPC-SMC cell sheet transplantation attenuated myocardial dysfunction, as evidenced by an increase in left ventricular ejection fraction (untreated vs cell sheet vs sham, $33.5 \%$ [IQR, 27.8\%-35.7\%] vs $45.9 \%$ [IQR, $43.6 \%-48.4 \%$ ] vs $59.3 \%$ [IQR, $58.8 \%-63.5 \%], P=.001)$ and decreases in left ventricular dimensions.

Conclusions: The bone marrow-derived, spatially arranged SMC-EPC bi-level cell sheet is a novel, multilineage cellular therapy obtained from a translationally practical source. Interactions between SMCs and EPCs augment mature neovascularization, limit adverse remodeling, and improve ventricular function after myocardial infarction. (J Thorac Cardiovasc Surg 2017;154:955-63)

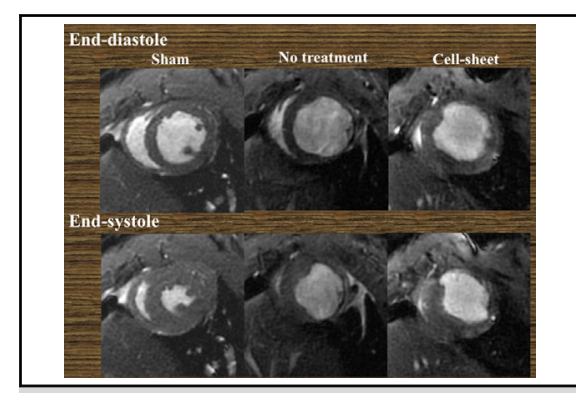

Endothelial progenitor cell-smooth muscle cell cell sheets enhance postinfarction ventricular function.

\section{Central Message}

An endothelial progenitor cell-smooth muscle cell construct-derived entirely from bone marrow cells-augments neovascularization and attenuates postinfarction myocardial remodeling.

\section{Perspective}

Endogenous effectors of myocardial repair are likely not limited to a single cell type. Tissue engineered cell sheets offer the potential to exploit natural interactions between various cell types. Here, we demonstrate that co-administration of endothelial progenitor cells and smooth muscle cells as an intact construct significantly improves endogenous mechanisms of myocardial repair, and these cells are obtainable from a translationally relevant source.

See Editorial Commentary page 964.

See Editorial page 951.
Despite medical and surgical advances, heart disease is the leading cause of death in the United States. Better characterization of stem cell lineages and endogenous effectors

\footnotetext{
From the a Department of Cardiothoracic Surgery, Stanford University School of Medicine, Stanford, Calif; and ${ }^{\mathrm{b}}$ Department of Cardiovascular Surgery, Osaka University Graduate School of Medicine, Osaka City, Japan.

This study was supported by the National Institutes of Health Grant 1R01HL08931501 (to Y.J.W.); American Heart Association Great Rivers Affiliate Postdoctoral Fellowship co-sponsored by the Claude R. Joyner Fund for Young Medical Researchers (\#12POST12060567) (to Y.S.); Uehara Memorial Foundation for Research Fellow, Japan (to Y.S.).

Read at the 96th Annual Meeting of The American Association for Thoracic Surgery, Baltimore, Maryland, May 14-18, 2016.
}

of myocardial repair have burgeoned interest in the use of cell therapies to improve left ventricular (LV) function in patients with advanced heart disease.

Received for publication May 26, 2016; revisions received April 8, 2017; accepted for publication April 12, 2017; available ahead of print June 23, 2017

Address for reprints: Y. Joseph Woo, MD, Department of Cardiothoracic Surgery, Falk Cardiovascular Research Center, Stanford University School of Medicine, Stanford, CA 94305 (E-mail: joswoo@stanford.edu).

0022-5223/\$36.00

Copyright (C) 2017 by The American Association for Thoracic Surgery http://dx.doi.org/10.1016/j.jtcvs.2017.04.081 


\section{Abbreviations and Acronyms \\ DMEM = Dulbecco's Modified Eagle's Medium \\ $\mathrm{ECM}=$ extracellular matrix \\ $\mathrm{EPC}=$ endothelial progenitor cells \\ FBS = fetal bovine serum \\ $\mathrm{LAD}=$ left anterior descending coronary artery \\ $\mathrm{LV} \quad=$ left ventricular \\ MSC = mesenchymal stem cells \\ MRI = magnetic resonance imaging \\ SMA $=$ smooth muscle actin \\ SMC $=$ smooth muscle cells}

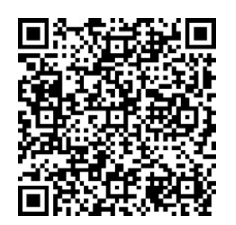

Tissue engineering is an essential component of developing effective regenerative therapies. ${ }^{5}$ In the past decade, tissue-engineered cell and scaffold therapies have been investigated widely, and several products are now commercially available. Scaffold-based tissue engineering is particularly popular and includes technologies such as biodegradable scaffolds, ${ }^{6}$ decellularized tissues, ${ }^{7}$ hydrogel and cell mixtures, ${ }^{8}$ bioprinting, ${ }^{9}$ and fiber-based tissue engineering. ${ }^{10}$ Our group has also used scaffold-free technology for cell-sheet engineering. ${ }^{11}$ The cell sheet is created on and removed from a specialized dish that is grafted covalently with a temperature-responsive polymer-poly ( $N$-isopropylacrylamide)—which undergoes an enzyme-free transformation from hydrophobic to hydrophilic by simply lowering the temperature. ${ }^{12}$ Thus, the specialized dishes permit fabrication of 3-dimensional tissues from densely adherent cells, without an artificial scaffold or enzymatic digestion. Cell sheets are manipulated easily and have a unique ability to integrate within native tissue; they retain cell-cell junctions as well as the extracellular matrix (ECM) deposited on the basal surface of cell sheet. ${ }^{4}$

We previously demonstrated that engineered cell sheets with smooth muscle cells (SMCs) and endothelial progenitor cells (EPCs) harnessed natural interactions between EPCs and SMCs, created structurally mature, functional microvasculature, and induced functional recovery of distressed myocardium. ${ }^{1}$ However, cell-sheet SMCs were obtained from the thoracic aorta and therefore precluded clinical translation. ${ }^{1}$ To resolve this problem, we have noted that bone marrow-derived mesenchymal stem cells (MSCs) have shown potential to differentiate into various cell types, including SMCs. ${ }^{2}$ Given that the ECM is a powerful regulator of SMC phenotypic modulation, ${ }^{3}$ we demonstrated that fibronectin helped guide differentiation of MSCs into SMCs while simultaneously preserving cellular proliferative capacity. ${ }^{4}$

In this study, we hypothesized that joint delivery of EPCs and SMCs in a novel, totally bone marrow-derived cell sheet will mimic the native architecture of a mature blood vessel and act as a supratherapeutic angiogenic construct to limit post infarction ventricular remodeling.

\section{MATERIALS AND METHODS \\ Isolation of MSCs and EPCs}

Bone marrow mononuclear cells were isolated from the long bones of male Wistar rats (8 weeks old, 250-300 g; Charles River Laboratories, Worchester, Mass), filtered through a $40-\mu \mathrm{m}$ Falcon cell strainer (Corning Inc, Corning, NY), and centrifuged at $300 \mathrm{~g}$ for 7 minutes. Red blood cells were excluded by the use of $1 \times$ RBC lysis buffer (\#00-4337-57; eBioscience/ Thermo Fisher Scientific, Waltham, Mass) for 10 minutes at $4^{\circ} \mathrm{C}$. Remaining cells were cultured in a medium with Dulbecco's Modified Eagle's Medium (DMEM; \#11995-040; Gibco Laboratories, Gaithersburg, Md) containing 10\% fetal bovine serum (FBS; Sigma-Aldrich, ST Louis, $\mathrm{Mo}$ ) and gentamicin on noncoated culture dishes for 24 hours at $37^{\circ} \mathrm{C}$. After incubation, the adherent cells were washed and then cultured in a medium with DMEM containing 10\% FBS and gentamicin. A purified population of MSCs was obtained 10 to 14 days after the initiation of culture. MSCs were identified in accordance with the criteria of the International Society for Cellular Therapy ${ }^{2}$; specifically, expression of CD 105, CD73, and CD 90, as well as absent expression of CD45 and CD34 were required. $^{4}$

EPCs were isolated and cultured as previously described. ${ }^{1}$ In summary, bone marrow mononuclear cells were isolated from the long bones of Wistar rats by density gradient centrifugation with Histopaque 1083 (SigmaAldrich) and cultured in endothelial basal medium-2 supplemented with EGM-2 SingleQuots (Lonza, Basel, Switzerland) containing human epidermal growth factor, 5\% FBS, vascular endothelial growth factor, basic human fibroblast growth factor, recombinant human long R3 insulin-like growth factor-1, ascorbic acid, and gentamicin on vitronectin (V0132-50VG; Sigma-Aldrich)-coated dishes. The combination of endothelium-specific media and the removal of nonadherent bone marrow mononuclear cells were intended to select for the EPC phenotype. The EPC phenotype was confirmed by expression of $\mathrm{CD} 31$, as previously described. ${ }^{4}$

\section{Transdifferentiation of MSCs Into SMCs}

Primary rodent MSCs were transferred and cultured in a medium with DMEM and $10 \%$ FBS on 60-mm culture dishes coated with fibronectin (FN group; BD Biosciences, San Jose, Calif) at $37^{\circ} \mathrm{C}$ in a humidified atmosphere of $5 \% \mathrm{CO}_{2}$ in air. Primary MSCs also were included in this study (control group). The cell number of the primary seeded MSCs was 4 to $6 \times 10^{3} / \mathrm{cm}^{2}$ for each plate. MSC growth medium was used as the nutrient medium, and all media were exchanged every 48 to 72 hours. $^{4}$

\section{Nanovolume Capillary Electrophoresis-Based Protein Analysis of Cultured Cells}

Nanovolume capillary electrophoresis-based protein analysis was performed on the cultured MSCs and SMCs with Wes (ProteinSimple Inc, San Jose, Calif). Tissue homogenates from cultured cells were prepared with Halt Protease Inhibitor Single-Use Cocktail (Thermo Fisher Scientific) diluted in T-PER Tissue Protein Extraction Reagent (Thermo Fisher 
Scientific). The protein concentration for the lysate was estimated by BioRad Protein Assay Dye Reagent Concentrate (Bio-Rad Laboratories, Inc, Hercules, Calif).

For each analysis, we applied as little as $2 \mathrm{pg}$ of protein to the prefilled plate according to the manufacturer's instructions. A charge-based separation (isoelectric focusing) was performed in a $5 \mathrm{~cm}$-long, $100-\mu \mathrm{m}$ inner diameter capillary cartridge. After separation and within-capillary immobilization, specific antibodies were applied to detect proteins of interest. Antibodies included rabbit polyclonal anti-alpha smooth muscle actin (SMA) antibody (ab5694, 1:50; Abcam), rabbit polyclonal anti-SM22 alpha antibody (ab14106, 1:50; Abcam), rabbit monoclonal anti-caldesmon antibody (ab32330, 1:50; Abcam), or vinculin (ab129002, 1:500; Abcam). To assess the intensity of bands for these proteins semiquantitatively, densitometric analysis was performed with Compass software (Lexington, Mass). The intensity level of detected protein bands was standardized by dividing by the intensity level of vinculin.

\section{Creation of Bi-Level Cell Sheets}

SMCs were plated at a density of $1.5 \times 10^{5} / \mathrm{cm}^{2}$ in a $35-\mathrm{mm}$ Upcell dish-which is grafted with temperature-responsive polymers (CellSeed, Tokyo, Japan)—and then cultured in EPC-specific medium. After 24 hours of culture at $37^{\circ} \mathrm{C}$ and $5 \% \mathrm{CO}_{2}$, EPCs were added at an equivalent density of $1.5 \times 10^{5} / \mathrm{cm}^{2}$ onto the Upcell dish, which already was confluent with SMCs. Each dish was transferred to room temperature for 30 minutes after an additional 24 hours in culture; a confluent bi-level cell sheet, made of EPCs and transdifferentiated SMCs, was then spontaneously detached as an intact cell sheet from the UpCell dish.

\section{Immunohistological Assessment of Bi-Level Cell Sheets}

The cell sheet, which consisted of EPCs and SMCs transdifferentiated from MSCs, was embedded in an optimum cutting temperature compound for $10-\mu \mathrm{m}$-thick sections. Cryosections were stained with rabbit polyclonal anti-alpha SMA antibody (ab5694, 1:100; Abcam) and mouse monoclonal anti-CD31 antibody (ab64543, 1:00; Abcam) to assess characteristics of the bi-level cell sheet.

\section{Rat Model of Ischemic Cardiomyopathy and Cell Sheet Transplantation}

Female Wistar rats (8 weeks old, 250-300 g; Charles River Laboratories) were anesthetized with isoflurane, endotracheally intubated with a 19-gauge catheter, and mechanically ventilated (Hallowell EMC, Pittsfield, Mass). Anesthesia was maintained by inhalation of $2.0 \%$ isoflurane. The proximal left anterior descending coronary artery (LAD) of Wistar rats was occluded permanently via a left thoracotomy approach. This produced a consistent and reproducible myocardial infarction encompassing $35 \%$ to $40 \%$ of the left ventricle. ${ }^{1}$ Within 5 minutes after LAD ligation, each rat was allocated: those that underwent co-cultured cell sheet transplantation (cell sheet group, $\mathrm{n}=9$ ) and those that underwent no treatment (untreated control group, $\mathrm{n}=12$ ). For comparative purposes, rats receiving a sham operation also were studied as a positive control (sham surgery control group, $\mathrm{n}=7$ ). In the cell sheet group, the co-cultured bi-level cell sheet, which consisted of $1.5 \times 10^{5} / \mathrm{cm}^{2}$ EPCs and $1.5 \times 10^{5} / \mathrm{cm}^{2} \mathrm{SMCs}$, was placed on the epicardium covering the ischemic area. Animals were then kept in temperature-controlled individual cages for 4 weeks. The rats were euthanized 4 weeks after surgery by intravenous injection of 2 $\mathrm{mEq} / \mathrm{kg}$ potassium chloride solution under terminal anesthesia, and the heart was excised.

\section{MRI Assessment of Cardiac Function}

Electrocardiogram-gated cardiac magnetic resonance imaging (MRI) was performed with a preclinical 7T (MR901 Discovery) horizontal bore scanner (Agilent, Santa Clara, Calif) with a shielded gradient system $(600 \mathrm{mT} / \mathrm{m}) 1$ to 3 days before and 4 weeks after myocardial infarction (cell sheet, $\mathrm{n}=9$; no treatment, $\mathrm{n}=12$; sham surgery, $\mathrm{n}=7$ ). Animals were anesthetized with $3.0 \%$ inhaled isoflurane and placed onto an animal cradle in prone position. Animals were kept at $37 \pm 0.4^{\circ} \mathrm{C}$ (during image acquisition) via an air heating system while oxygen and anesthetics ( $1 \%-2 \%$ isoflurane) were supplied via a nose cone $(0.5 \mathrm{~L} / \mathrm{min})$. Data acquisition was performed with a 4-channel phased array receive-only surface coil (Rapid MR International, Columbus, Ohio) placed around the heart and centered in a decoupled 72-mm transmit/receive volume coil (Agilent). Long- and short-axis scout images were acquired to define the 2- and 4-chamber long-axis views. The cine long-axis views were used to define the short-axis orientation. A prospectively double-gated (electrocardiography and respiration) spoiled gradient echo sequence was used to acquire cine cardiac images with the following parameters for standard cine acquisitions: echo time 1.5 milliseconds, repetition time 6 to 8 milliseconds, flip angle $15^{\circ}$, slice thickness $1 \mathrm{~mm}$, no slice separation, field of view $50 \times 50 \mathrm{~mm}^{2}$, matrix size $192 \times 192$, number of signal averages 1 for short-axis and 2 for long-axis. Twenty cine frames were recorded to cover the cardiac cycle. A single short-axis slice was obtained in approximately 45 seconds, leading to a total scan time of 11 to 13 minutes, covering the heart from base to apex (14-15 slices).

Imaging parameters for these acquisitions were as follows: echo time 1.4 milliseconds, repetition time one breathing interval, inversion time 280 to 370 milliseconds, flip angle $90^{\circ}$, slice thickness $1 \mathrm{~mm}$, no slice separation, field of view $40 \times 40 \mathrm{~mm}^{2}$, matrix size $192 \times 192$, number of signal averages 2 , and views per segment 2 . The acquisition time was roughly 1 minute per slice. The imaging protocol for one rat typically required 45-50 minutes. All images from one animal were combined into a dataset, randomized, and anonymized. Data analysis was performed with the semiautomatic segmentation software Segment (Medviso AB, Lund, Sweden) as previously described. ${ }^{13}$

Routine parameters of LV geometry and function, such as LV enddiastolic volume, LV end-systolic volume, and LV ejection fraction, were measured according to the slice summation method using LV functional analysis software (OsiriX MD Imaging Software, version 3.0; Pixmeo SARL, Bernex, Switzerland). All analyses were performed by a single investigator in a group-blinded fashion.

\section{Assessment of Mature Vessel Formation}

Four weeks postmyocardial infarction, all rat hearts were dissected and embedded in an optimum cutting temperature compound for $10-\mu \mathrm{m}$ thick sections. Heart cryosections were stained with sheep polyclonal anti-von Willebrand factor antibody (ab8822, 1:100; Abcam) and rabbit polyclonal anti-alpha SMA antibody (ab5694, 1:100; Abcam) to assess mature vessel density. Mature vessel density was calculated as the number of positively stained vessels in 5 randomly selected fields within the peri-infarct border zone, per heart. Cell nuclei were counterstained with 4,6-Diamidino-2-phenylindole (DAPI; Vector Laboratories, Burlingame, Calif). Images were acquired with fluorescence microscopy (Leica, Wetzlar, Germany) and ImageJ software (https://imagej.nih.gov/ij/) was used for quantitative morphometric analyses.

\section{Assessment of Myocardial Fibrosis}

Four weeks after the treatment, the hearts were explanted, embedded in optimum cutting temperature compound, and cut into $10-\mu \mathrm{m}$ thick sections. Masson's trichrome staining was performed to assess cardiac fibrotic extension. The fibrotic burden was calculated as the degree of fibrosis along the circumferential length of the LV at the mid-LV level, which was defined as the mid-point between the base and apex of the $\mathrm{LV}$ ( $\mathrm{n}=4$ per group).

\section{Statistical Analysis}

Continuous variables are expressed as the median and interquartile range (IQR). Because of small sample sizes, comparisons between 2 groups were made with the Wilcoxon-Mann-Whitney $U$ test. For comparisons between 3 groups, we used the Kruskal-Wallis test followed by post hoc pairwise Wilcoxon-Mann-Whitney $U$ tests. The multiplicity in pairwise 


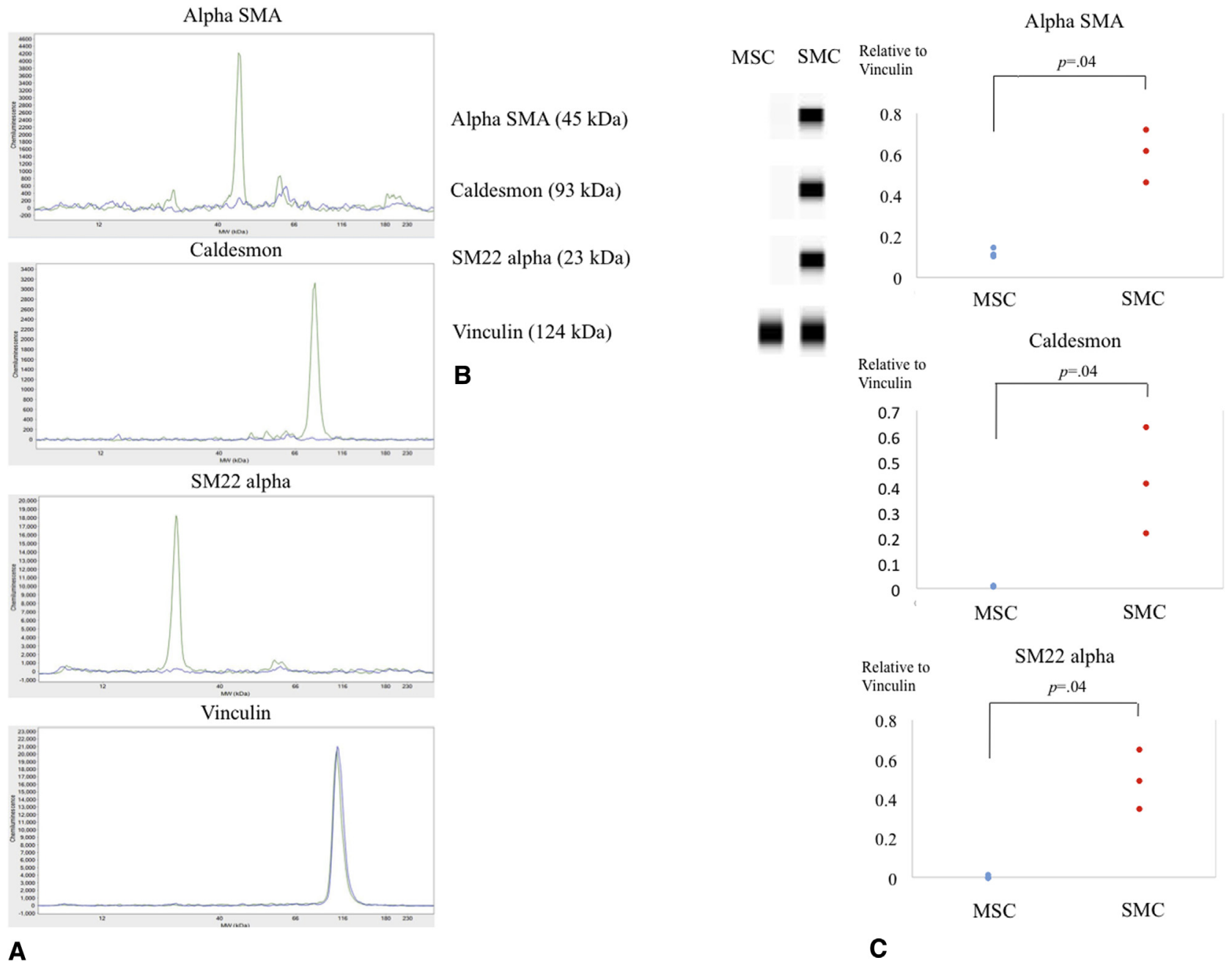

FIGURE 1. Nanovolume capillary electrophoresis-based protein analysis was performed on cultured MSCs and transdifferentiated SMCs $($ MSC, $n=3$; $\mathrm{SMC}, \mathrm{n}=3$ ). A, Electropherogram and system software-generated peak area and molecular weight data linear analysis for alpha SMA, SM22 alpha, and caldesmon between MSCs and SMCs. Green indicates SMC; blue, MSC. B, Representative immunoblots for alpha SMA, SM22 alpha, and caldesmon between MSC and SMC. C, Transdifferentiated SMCs demonstrated greater protein concentrations of alpha SMA, SM22 alpha, and caldesmon than MSCs. The intensity level of detected protein bands was standardized by dividing by the intensity level of vinculin. SMA, Smooth muscle actin; MSCs, mesenchymal stem cells; SMCs, smooth muscle cells.

comparisons was corrected by the Bonferroni procedure. For categorical variables, 2 groups were compared with the Fisher exact test. A $P$ value less than .05 was considered statistically significant. All calculations were performed with JMP 9.0 (SAS Institute Inc, Cary, NC).

\section{Animal Care and Biosafety}

Wistar rats were obtained from Charles River Laboratories. Food and water were provided ad libitum. This investigation conformed with the Guide for the Care and Use of Laboratory Animals published by the US National Institutes of Health (National Institutes of Health Publication No. 85-23, revised 1996) and was approved by the Institutional Animal Care and Use Committee of Stanford University (protocol 28921).

\section{RESULTS}

\section{Characterization of MSCs and Transdifferentiated SMCs}

Proportions of proteins indicative of the SMC phenotype were examined by nanovolume capillary electrophoresis- based protein analysis. MSCs were isolated from bone marrow and transdifferentiated via culture on fibronectincoated dishes. Expression levels of alpha SMA (MSC vs SMC, 0.11 [IQR, 0.10-0.13] vs 0.62 [IQR, 0.54-0.67], $P=.04$ ), SM22 (MSC vs SMC, 0.010 [IQR, 0.010-0.013] vs 0.490 [IQR, 0.419-0.570], $P=.04$ ), and caldesmon (MSC vs SMC, 0.005 [IQR, 0.005-0.007] vs 0.413 [IQR, $0.315-0.524], P=.04$ ) were significantly elevated in transdifferentiated MSCs compared with that of primary MSCs (Figure 1).

\section{Characterization of Bi-Level Cell Sheets}

The bi-level cell sheet maintained alpha SMA-positive SMCs and CD31-positive EPCs in separate layers in vitro (Figure 2, A). 

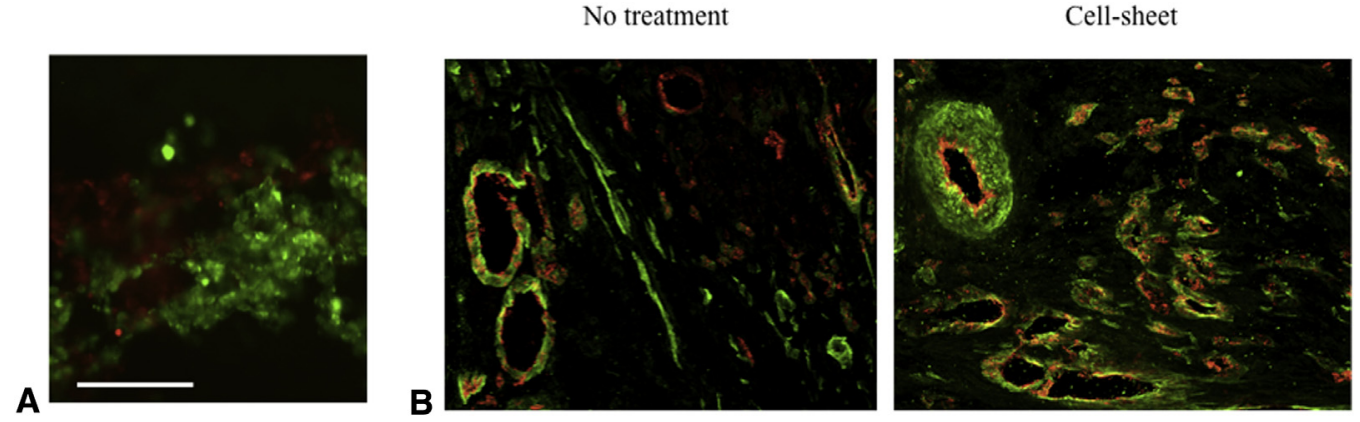

Mature vessel density $\left(/ \mathrm{mm}^{2}\right)$

$$
p=.08
$$

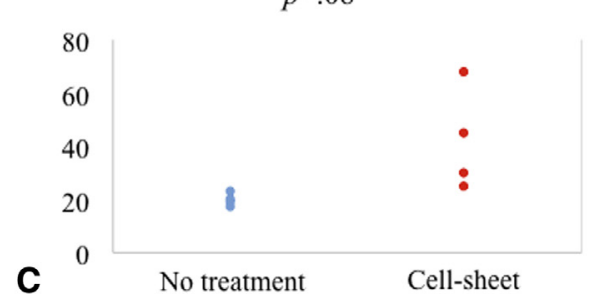

FIGURE 2. A, The bi-level cell sheet maintained alpha SMA-positive SMCs and CD31 positive EPCs in separate layers in vitro. Green indicates alpha SMA; red, CD31. White bar $=100 \mu \mathrm{m}$. B, Representative images demonstrating vWF and alpha SMA staining of border zone myocardium for cell sheettreated and untreated groups. Green indicates alpha SMA; red, vWF. C, Quantification of arterial density. Mature artery density was increased in the cell sheet-treated group compared with the untreated group.

\section{Coadministered EPCs and SMCs Likely Enhance Intramyocardial Arterial Density}

Four weeks postinfarction, a larger number of arteries (von Willebrand factor/alpha SMA-positive blood vessels) were detected in the cell sheet-treated myocardium compared with that of the untreated group (untreated vs cell sheet, $18 / \mathrm{mm}^{2}$ [IQR, $16 / \mathrm{mm}^{2}-29 / \mathrm{mm}^{2}$ ] vs $37 / \mathrm{mm}^{2}$ $\left.\left[25 / \mathrm{mm}^{2}-120 / \mathrm{mm}^{2}\right], P=.08\right)$, but this result only approached our threshold for statistical significance (Figure 2, $B$ and $C$ ).

\section{EPC-SMC Cell Sheets Attenuate Cardiac Fibrosis}

Four weeks after myocardial infarction, cell sheet therapy significantly attenuated the extent of cardiac fibrosis compared with that of the untreated group, as demonstrated by Masson's trichrome staining (untreated vs cell sheet, 198 degrees [IQR, 151-246 degrees] vs 103 degrees [IQR, 92113 degrees], $P=.04$ ) (Figure 3); no fibrosis was noted in the sham group.

\section{EPC-SMC Cell Sheets Improve Cardiac Function and Attenuate LV Remodeling}

The effects of co-cultured bi-level cell sheet transplantation on cardiac function were assessed in a rat model of ischemic cardiomyopathy. Compared with sham surgery, permanent ligation of the LAD in untreated animals significantly decreased LV ejection fraction with concomitant increases in LV end systolic and end diastolic volume 4 weeks after infarction, all characteristics of chronic ischemic heart failure. However, EPC-SMC cell-sheet transplantation attenuated myocardial dysfunction, as evidenced by an increase in LV ejection fraction (untreated vs cell sheet vs sham, 33.5\% [IQR, 27.8\%-35.7\%] vs $45.9 \%$ [IQR, $43.6 \%-48.4 \%]$ vs $59.3 \%$ [IQR, $58.8 \%-63.5 \%$ ], $P=.001)$ and induced significant reverse LV remodeling, as evidenced by decreases in LV dimensions (LV enddiastolic volume, untreated vs cell sheet vs sham, $688 \mu \mathrm{L}$ [IQR, 604-787 $\mu \mathrm{L}]$ vs $586 \mu \mathrm{L}$ [IQR, 505-661 $\mu \mathrm{L}$ ] vs $416 \mu \mathrm{L}$ [IQR, 399-469 $\mu \mathrm{L}$ ], $P=.001 ; \mathrm{LV}$ end-systolic volume, untreated vs cell sheet vs sham, $446 \mu \mathrm{L}$ [IQR, 374$557 \mu \mathrm{L}$ ] vs $317 \mu \mathrm{l}$ [IQR, 266-372 $\mu \mathrm{L}$ ] vs $151 \mu \mathrm{L}$ [IQR, $147-191 \mu \mathrm{L}], P=.001$ ) (Figure 4).

\section{DISCUSSION}

Development of novel therapies that are clinically translatable is critical to transition research from the bench to the bedside. Although our previous EPC-SMC cell sheets were potent angiogenic constructs, use of the aorta as a source for SMC isolation precluded translation as a realistic therapy. Here, we demonstrate that MSCs may be encouraged toward the SMC phenotype simply by culturing bone marrow-derived MSCs on fibronectin-coated dishes. Then, a confluent co-cultured bi-level cell sheet made of isolated EPCs and transdifferentiated SMCs was engineered using cell sheet technology. The result was increased arterial density, as well as attenuated postinfarction myocardial dysfunction and remodeling.

We have reported that bi-level cell sheets, consisting of co-cultured SMCs and EPCs, create architecturally mature, 

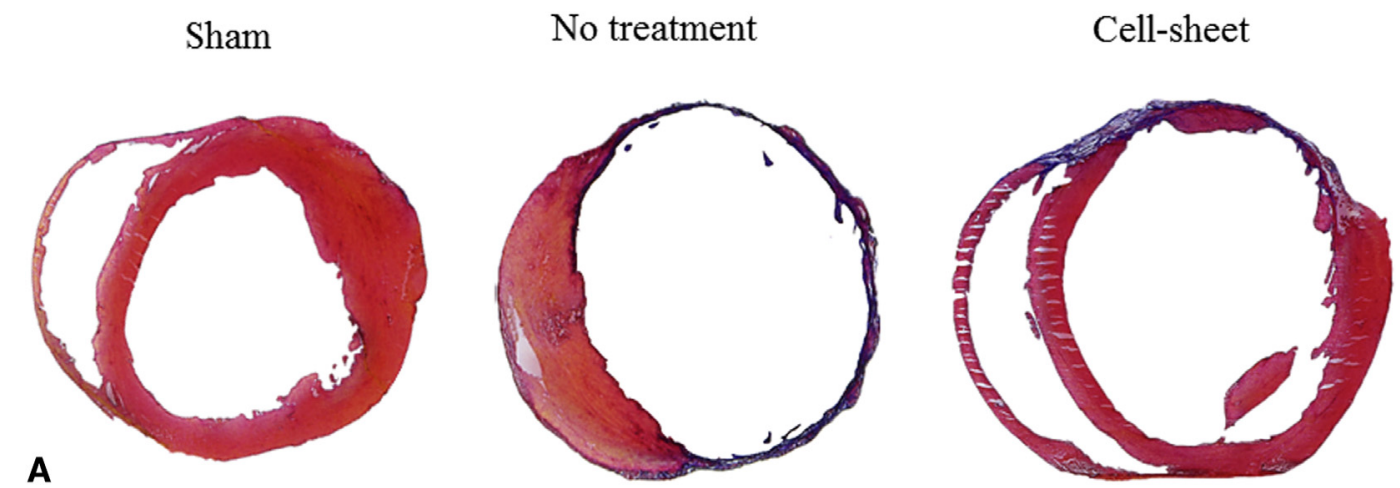

Cardiac fibrotic extension (degrees)

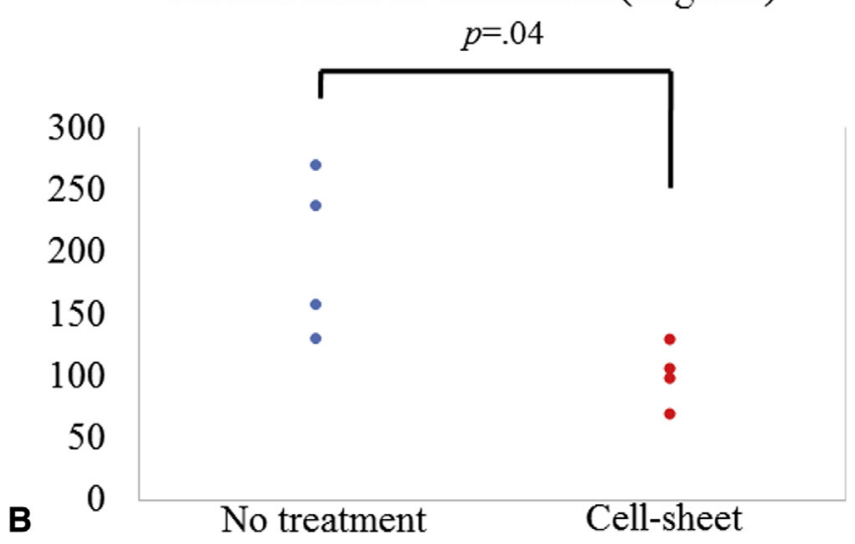

FIGURE 3. A, Representative Masson's trichrome staining of the heart for cell sheet-treated $(n=4)$, untreated $(n=4)$, and sham control ( $n=4)$ groups. B, Quantification of cardiac fibrotic extension. Fibrosis was suppressed significantly in the cell-sheet group compared with the untreated group.

functional microvasculature by maintaining the natural interactions between EPCs and SMCs, thereby enhancing myocardial function beyond that of cell injection therapy. ${ }^{1}$ Cell sheets deliver cells more effectively than intracoronary or intramyocardial injection because cell dispersion and myocardial injury are lessened. However, the ability to acquire the requisite cells in a less invasive and efficient fashion is crucial. To address this, the use of somatic adult stem cells (eg, MSCs) has been suggested for use in regenerative medicine. ${ }^{14}$ MSCs are an attractive autologous cell source for cell-based regenerative therapies due to their strong ability to proliferate and differentiate into various cell types, including chondrocytes, osteocytes, adipocytes, skeletal myoblasts, and cardiomyocytes. ${ }^{15,16}$ Moreover, MSCs can be isolated in a minimally invasive fashion from various tissues, such as the bone marrow, umbilical cord, amniotic fluid, peripheral blood, and adipose tissue. These concepts led us to examine MSCs as a potential source of SMCs for cell sheet engineering. By testing different types of ECM, we found that isolated MSCs can be reliably transdifferentiated into the SMC lineage-while simultaneously maintaining proliferative capacity- simply by culturing on commercially available fibronectin-coated dishes. ${ }^{4}$ Here, we provide evidence that the SMCs transdifferentiated from MSCs maintain their in vivo therapeutic capacity, an essential finding if such a therapy is to be translated to the clinical arena.

Tissue engineering therapeutics created from cells and scaffolds have been investigated widely. Cell sheet technology has been used to regenerate a number of damaged tissues. Several studies, including clinical trials, have been conducted to examine whether cell sheets effectively repair the cornea, epithelium, ${ }^{17}$ or heart. ${ }^{11,18}$ Confluent cells on a temperature responsive culture surface can be harvested as an intact and contiguous cell sheet by simply reducing the temperature without protease treatment. The significant features of the cell sheet are that cell-cell junctions and ECM components mediating cell adhesion are retained in the cell sheet without artificial scaffolds. In contrast, conventional cell harvesting using enzymatic digestion (eg, trypsin) disrupts all cell-cell junctions and adhesion proteins between cells. The EPC-SMC bi-level cell sheet retained regional morphologic differences between different cell types after mobilization from the UpCell dish. As determined by scanning electron microscopy, the SMC layer 

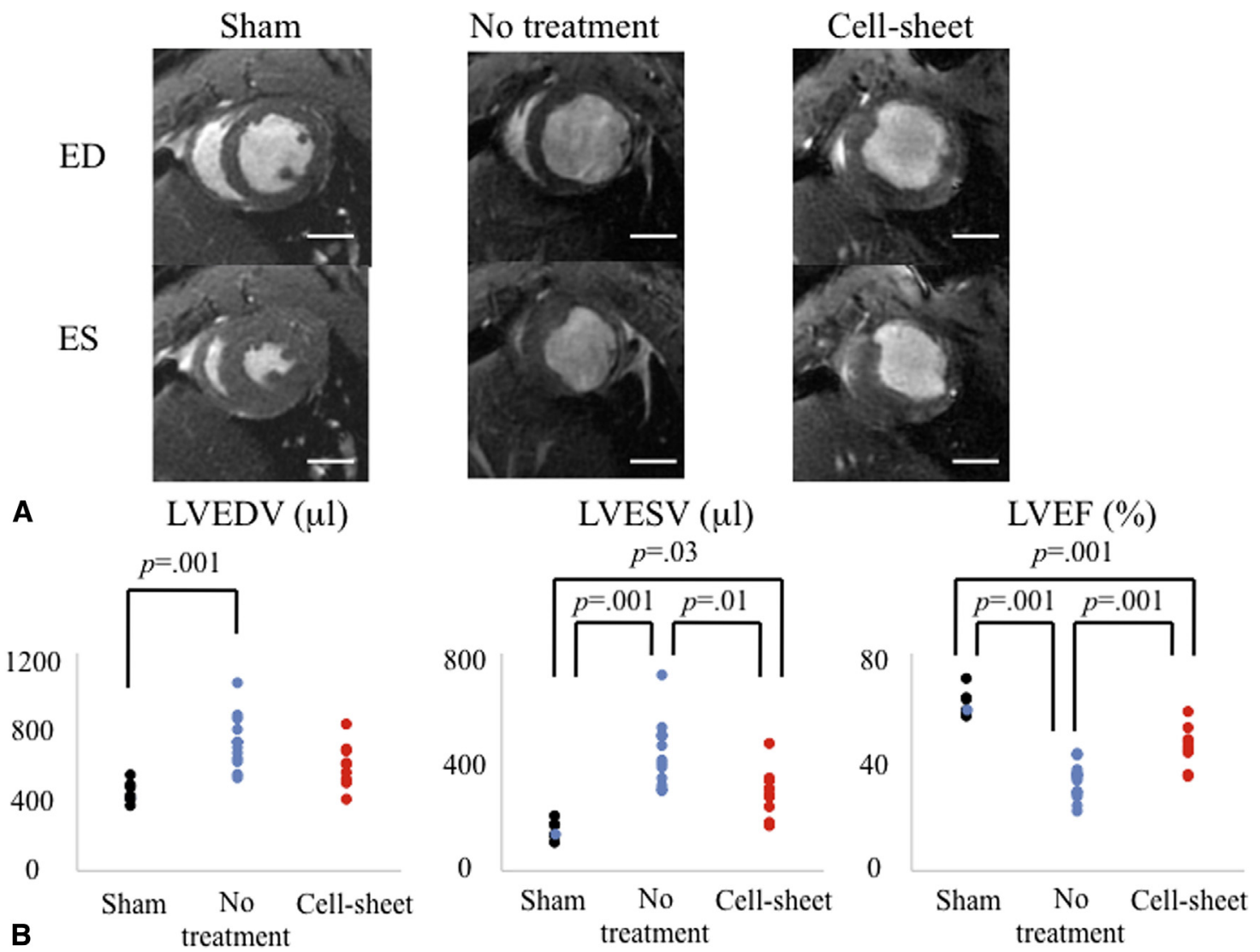

FIGURE 4. A, Representative cardiac magnetic resonance images at ED and ES phases for cell sheet-treated $(\mathrm{n}=9)$, untreated $(\mathrm{n}=12)$, and sham control $(\mathrm{n}=7)$ groups. Examinations were performed 4 weeks after myocardial infarction. B, Remarkable differences were noted between the 3 groups with respect to LVEDV, LVESV, and LVEF. $E D$, End-diastolic; $E S$, end-systolic; $L V E D V$, left ventricular end-diastolic volume; $L V E S V$, left ventricular end-systolic volume; $L V E F$, left ventricular ejection fraction.

contained spherical cells and the EPC layer formed a thin film-like monolayer. ${ }^{4}$

The mechanism by which the bone marrow-derived EPC-SMC bi-level cell sheet limited adverse ventricular remodeling and improved cardiac function is complex. Its contribution to the creation of mature arteries seems to be essential; we have demonstrated previously with cell fate tracking that a bi-level cell sheet, which contained EPCs and SMCs in separate layers, directly incorporates into perfused vasculature and remains present 4 weeks postinfarction. ${ }^{1}$ In the present study, cell sheet transplantation increased arterial density. One possible explanation is that maintenance of the interactions between EPCs and their physiologic support cell, SMCs, augments arterialization and in turn limits adverse remodeling after myocardial infarction.

For more than a decade, echocardiography has been used ubiquitously to measure LV function and volume in rodents with ischemic cardiomyopathy. However, results are based on geometric assumptions that limit data accuracy. Furthermore, assumptions within the calculations of parameters of cardiac function are typically violated after a myocardial infarction. Recent advances in cardiac MRI have resulted in a shorter acquisition time and improved image quality. As such, cardiac MRI is now considered the reference standard for accurate assessment of LV geometry and volume in humans. Cardiac MRI also has been used to evaluate cardiac function in small animals. ${ }^{13}$ It provides excellent temporal and spatial resolution, it is very accurate, and it facilitates reproducible quantitative measurements of cardiac structure and function. ${ }^{19}$ In our study, cardiac MRI illustrated postinfarction, eccentric adverse ventricular remodeling in both cell sheet-treated and untreated negative control groups. However, EPC-SMC cell sheet transplantation significantly enhanced ventricular function and attenuated ventricular remodeling compared with that of untreated controls. Because we used an acute myocardial infarction model in our study and only imaged the heart at a single time point, we cannot determine whether the observed changes in cardiac function and LV dimensions postinfarct were the result of reverse remodeling or simply mitigation of adverse remodeling. 
Considering the time required to isolate, cultivate, transdifferentiate, and manipulate cells in vitro, this treatment strategy for acute myocardial infarction is not yet directly applicable to the clinical arena. However, such a construct may be a potential candidate for allogeneic therapy. Further investigation into the utility of these cell sheets in treating chronic ischemic cardiomyopathy and diabetic cardiomyopathy are ongoing, as is an assessment of the duration of transplanted cell survival. Finally, whether bi-layer cell sheets are incrementally more effective than EPC, SMC, or MSC monolayer cell sheets requires further testing.

In conclusion, the bone marrow-derived, anatomically oriented bi-level cell sheet made of isolated EPCs and transdifferentiated SMCs is a novel, multilineage cellular therapy obtained from a translationally practical source. Delivery of a tissue-engineered construct that maintains important interactions between EPCs and SMCs enhances mature neovascularization within border zone myocardium, tempers postinfarction adverse remodeling, and strengthens ventricular function.

\section{Webcast}

You can watch a Webcast of this AATS meeting presentation by going to: http://webcast.aats.org/2016/Video/ Tuesday/05-17-16_Ballroom_IV_0740_Shudo-800.mp4.

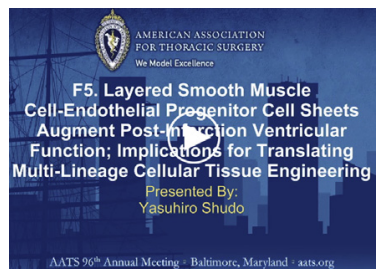

\section{Conflict of Interest Statement}

Authors have nothing to disclose with regard to commercial support.

\section{References}

1. Shudo Y, Cohen JE, MacArthur JW, Atluri P, Hsiao PF, Yang EC, et al. Spatially oriented, temporally sequential smooth muscle cell-endothelial progenitor cell bi-level cell sheet neovascularizes ischemic myocardium. Circulation. 2013; 128(11 suppl 1):S59-68.

2. Dominici M, Le Blanc K, Mueller I, Slaper-Cortenbach I, Marini F, Krause D, et al. Minimal criteria for defining multipotent mesenchymal stromal cells. The International Society for Cellular Therapy position statement. Cytotherapy. 2006;8:315-7.

3. Thyberg J, Hedin U, Sjolund M, Palmberg L, Bottger BA. Regulation of differentiated properties and proliferation of arterial smooth muscle cells. Arteriosclerosis. 1990;10:966-90.

4. Shudo Y, Cohen JE, Goldstone AB, MacArthur JW, Patel J, Edwards BB, et al. Transdifferentiation of mesenchymal stem cell into smooth muscle cell lineage; utility for clinical application from isolation to creation of cell-sheet. Cytotherapy. 2016;18:510-7.

5. Langer R, Vacanti JP. Tissue engineering. Science. 1993;260:920-6.

6. Atluri P, Trubelja A, Fairman AS, Hsiao PF, MacArthur JW, Cohen JE, et al. Normalization of post-infarct biomechanics utilizing a novel tissue engineered angiogenic construct. Circulation. 2013;128(26 Suppl 1):S95-104.
7. Neumann A, Sarikouch S, Breymann T, Cebotari S, Boethig D, Horke A, et al. Early systemic cellular immune response in children and young adults receiving decellularized fresh allografts for pulmonary valve replacement. Tissue Eng Part A. 2014;20:1003-11.

8. Cohen JE, Purcell BP, MacArthur JW Jr, Mu A, Shudo Y, Patel JB, et al. A bioengineered hydrogel system enables targeted and sustained intramyocardial delivery of neuregulin, activating the cardiomyocyte cell cycle and enhancing ventricular function in a murine model of ischemic cardiomyopathy. Circ Heart Fail. 2014;7:619-26.

9. Murphy SV, Atala A. 3D bioprinting of tissues and organs. Nat Biotechnol. 2014; 32:773-85.

10. Onoe H, Okitsu T, Itoh A, Kato-Negishi M, Gojo R, Kiriya D, et al. Metre-long cell-laden microfibers exhibit tissue morphologies and functions. Nat Mater. 2013;12:584-90.

11. Sawa Y, Miyagawa S, Sakaguchi T, Fujita T, Matsuyama A, Saito A, et al. Tissue engineered myoblast sheets improved cardiac function sufficiently to discontinue LVAS in a patient with DCM: report of a case. Surg Today. 2012;42:181-4.

12. Okano T, Yamada N, Sakai H, Sakurai Y. A novel recovery system for cultured cells using plasma-treated polystyrene dishes grafted with poly (N-iso-propylacrylamide). J Biomed Mater Res. 1993;27:1243-51.

13. Riegler J, Gillich A, Shen Q, Gold JD, Wu JC. Cardiac tissue slice transplantation as a model to assess tissue-engineered graft thickness, survival, and function. Circulation. 2014;130(11 suppl 1):S77-86.

14. Shudo Y, Miyagawa S, Ohkura H, Fukushima S, Saito A, Shiozaki M, et al. Addition of mesenchymal stem cells enhances the therapeutic effects of skeletal myoblast cell-sheet transplantation in a rat ischemic cardiomyopathy model. Tissue Eng Part A. 2013;20:728-39.

15. Jiang Y, Jahagirdar BN, Reinhardt RL, Schwartz RE, Keene CD, OrtizGonzalez XR, et al. Pluripotency of mesenchymal stem cells derived from adult marrow. Nature. 2002;418:41-9.

16. Prockop DJ. Marrow stromal cells as stem cells for non-hematopoietic tissues. Science. 1997;276:71-4.

17. Nishida K, Yamato M, Hayashida Y, Watanabe K, Yamamoto K, Adachi E, et al. Corneal reconstruction with tissue-engineered cell sheets composed of autologous oral mucosal epithelium. N Engl J Med. 2004;351:1187-96.

18. Shudo Y, Miyagawa S, Fukushima S, Saito A, Shimizu T, Okano T, et al. Novel regenerative therapy using cell-sheet covered with omentum flap delivers a huge number of cells in a porcine myocardial infarction model. $J$ Thorac Cardiovasc Surg. 2011;142:1188-96.

19. Shudo Y, Taniguchi K, Takeda K, Sakaguchi T, Funatsu T, Kondoh H, et al. Serial multidetector computed tomography assessment of left ventricular reverse remodeling, mass, and reginal wall stress after restrictive mitral annuloplasty in dilated cardiomyopathy. J Thorac Cardiovasc Surg. 2012;143(4 suppl):S43-7.

Key Words: tissue engineering, cell sheet, neovascularization, myocardial infarction, regeneration, stem cells

\section{Discussion}

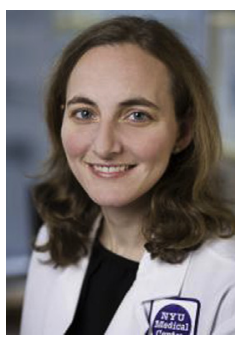

Dr L. Balsam (New York, NY). I would like to congratulate Dr Shudo and his colleagues for yet another contribution in the area of cell-based therapies postmyocardial infarction. To highlight one of the important aspects of your study, you have looked again for an autologous source for cells that could be clinically relevant. Your previous work using SMCs derived from the aorta has now been followed up with a study using an entirely bone marrow-derived group of cells.

In your previous work, you have demonstrated that there is incorporation of cells from your graft into the vasculature, and I want to ask you, in this current study have you gone to 
perform those experiments showing that this is not simply a paracrine effect that you are seeing with these cell sheets, how long do these sheets survive after you put them in the infarct milieu, and are these cells actually incorporating into these new blood vessels?

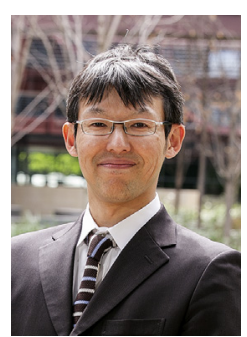

Dr Shudo (Stanford, Calif). That is an excellent question. Thank you for your comments. We performed the cell tracking experiments 4 weeks after cell-sheet implantation using the in a small animal acute myocardial infarction model. First, for the cell tracking, we did use a specific track system; we isolated EPCs from the a green fluorescent protein (GFP)positive female rat and then we got SMCs from the GFPnegative male rat, and then we created the bilateral bi-level cell sheet and implanted it onto the heart of female GFPnegative rat. The important meaning that GFP is supposed to be coming from the implanted EPC, and then the male, which means the $\mathrm{Y}$ chromosome, is supposed to be coming from the implanted SMC. We traced stained the GFP, Y chromosome, and then some components of the inner layer and outer layer of vessels.

We found noticed that the GFP positive and then the von Willebrand factor became positive in the inner layer, and also $\mathrm{Y}$ chromosome positive and then also alpha SMA positive outer layer, which means that implanted cell sheets directly contributed to the neovascularization in the host myocardium.
Dr Balsam. My last question really refers to the model that you used to look at the ability to repair or regenerate myocardium or vessels after infarct. You have chosen to apply these cell sheets immediately after an infarct, and as a clinician, I try to relate that to a clinical scenario. So, you have a patient who has just had a myocardial infarction, and the idea is to add a reparative strategy at the time of infarct. This is not necessarily so practical, particularly if major surgery is needed to apply the sheets. And, in addition, you describe that it takes 14 days to generate these SMCs derived from bone marrow.

So do you think you should be looking at this in a different model to make it clinically relevant?

Dr Shudo. Right. That is a good point. Bone marrow mononuclear cells were isolated and cultured for 10-14 days to obtain a purified MSC culture, and then it takes about 10 more days to successfully transdifferentiate into the SMC. So, in total it takes is 3 weeks to 4 weeks. That is a good point.

This study involved the small animal acute myocardial infarction model. I agree with your comments. We are now considering using a different disease model, like for example, a chronic myocardial infarction model; like induced myocardial infarction, 4 weeks after that implanted cell sheet, and then waiting 4 weeks, after that assess the cardiac function or something like that, or a different disease model, means heart failure, no ischemic cardiomyopathy model, or diabetes model. That is a good option. 